Article

\title{
On Convergence Rates of Some Limits
}

\section{Edward Omey ${ }^{1, *}$ and Meitner Cadena ${ }^{2}$}

1 Research Centre for Mathematics, Education, Econometrics and Statistics (MEES), Catholic University Leuven at Campus Brussels, Warmoesberg 26, 1000 Brussels, Belgium

2 Departamento de Ciencias Exactas, Universidad de las Fuerzas Armadas, Sangolqui 171103, Ecuador; meitner.cadena@gmail.com

* Correspondence: edward.omey@kuleuven.be

Received: 9 March 2020; Accepted: 17 April 2020; Published: 21 April 2020

Abstract: In 2019 Seneta has provided a characterization of slowly varying functions $L$ in the Zygmund sense by using the condition, for each $y>0, x\left(\frac{L(x+y)}{L(x)}-1\right) \rightarrow 0$ as $\quad x \rightarrow \infty$. Very recently, we have extended this result by considering a wider class of functions $U$ related to the following more general condition. For each $y>0, r(x)\left(\frac{U(x+y g(x))}{U(x)}-1\right) \rightarrow 0$ as $\quad x \rightarrow \infty$, for some functions $r$ and $g$. In this paper, we examine this last result by considering a much more general convergence condition. A wider class related to this new condition is presented. Further, a representation theorem for this wider class is provided.

Keywords: slowly varying; monotony in the Zygmund sense; class $\Gamma_{a}(g)$; self-neglecting function; convergence rates

\section{Introduction}

The notion of ultimately monotony introduced by Zygmund says that a function $U \geq 0$ is slowly varying if for each $\epsilon>0$ the function $x^{\epsilon} U(x)$ is ultimately increasing and $x^{-\epsilon} U(x)$ is ultimately decreasing ([1], p. 186). A different kind of slowly varying functions was defined by Karamata [2] known as simply the class of slowly varying functions (KSV). It is known that any ZSV function is a KSV function (see [1], p. 186 and, e.g., [3], p. 49).

Recently, Seneta [4] found that the slowly varying functions $L$ in the sense of Zygmund are characterized by the relation:

$$
\lim _{x \rightarrow \infty} x\left(\frac{L(x+y)}{L(x)}-1\right)=0, \forall y .
$$

More recently, Omey and Cadena's [5] functions extended the results of Seneta, and they considered functions for which the following relation holds:

$$
\lim _{x \rightarrow \infty} r(x)\left(\frac{L(x+y g(x))}{L(x)}-1\right)=0, \quad \forall y .
$$

Here, the function $g(x)$ is self-neglecting (notation: $g \in S N$ ) and $r$ is in the class $\Gamma_{0}(g)$ with $r(x) \rightarrow \infty$. The class $\Gamma_{0}(g)$ is deeply studied in [6]. Recall that $g \in S N$ if it satisfies

$$
\lim _{x \rightarrow \infty} \frac{g(x+y g(x))}{g(x)}=1,
$$

locally uniformly in $y$. In addition, recall that, for $g \in S N$, we have $f \in \Gamma_{\alpha}(g)$ if $f$ satisfies

$$
\lim _{x \rightarrow \infty} \frac{f(x+y g(x))}{f(x)}=e^{\alpha y}, \forall y .
$$


Now, we study more general relations of the form

$$
\lim _{x \rightarrow \infty} r(x)\left(\frac{U(x+y g(x))}{U(x)}-e^{\alpha y}\right)=\theta(y), \quad \forall y,
$$

where we assume that the convergence is l.u. in $y$. As before, we assume that $r \in \Gamma_{0}(g), r(x) \rightarrow \infty$ and that $g \in S N$.

Throughout this paper, we use the notation $f(x) \sim g(x)$ for representing $f(x) / g(x) \rightarrow 1$ as $x \rightarrow \infty$.

We study in detail the two cases: $\alpha=0$ and $\alpha \neq 0$. The case $\alpha=0$ can be considered as the class $S N$ with a rate of convergence in the definition. This case is presented in the following section. The case where $\alpha \neq 0$ can be considered as the class $\Gamma_{\alpha}(g)$ with a rate of convergence in the definition. This case is presented in Section 3. For each case, characterizations of the involved functions are provided. Concluding remarks are presented in the last section.

\section{The Case $\alpha=0$}

\subsection{The Limit Function}

Suppose that $U, g, r>0$ are measurable functions and suppose that the following relation holds:

$$
\lim _{x \rightarrow \infty} r(x)\left(\frac{U(x+y g(x))}{U(x)}-1\right)=\theta(y),
$$

and we assume that Equation (1) holds locally uniformly in $y$. As before, we assume that $r(x) \rightarrow \infty$, $r \in \Gamma_{0}(g)$ and that $g \in S N$.

Clearly, Equation (1) holds if and only if

$$
\lim _{x \rightarrow \infty} r(x)(W(x+y g(x))-W(x))=\theta(y),
$$

where $W(x)=\log U(x)$.

Now, we replace $x$ by $x=t+z g(t)$. Note that $g(t) / t \rightarrow 0$ so that $x / t \rightarrow 1$ l.u. in $z$. We find

$$
\lim _{t \rightarrow \infty} r(t+z g(t))(W(t+z g(t)+y g(t+z g(t)))-W(t+z g(t)))=\theta(y) .
$$

Using $r \in \Gamma_{0}(g)$, we have

$$
\lim _{t \rightarrow \infty} r(t)(W(t+z g(t)+y g(t+z g(t)))-W(t+z g(t)))=\theta(y),
$$

and then it follows that

$$
\lim _{t \rightarrow \infty} r(t)(W(t+z g(t)+y g(t+z g(t)))-W(t))=\theta(y)+\theta(z) .
$$

Now, we have

$$
W(t+z g(t)+y g(t+z g(t)))-W(t)=W\left(t+\left(z+y \frac{g(t+z g(t))}{g(t)}\right) g(t)\right)-W(t) .
$$

Using l.u. convergence, we obtain that

$$
\lim _{t \rightarrow \infty} r(t)(W(t+z g(t)+y g(t+z g(t)))-W(t))=\theta(y+z) .
$$

We conclude that

$$
\theta(z+y)=\theta(z)+\theta(y)
$$


and (since $\theta$ is measurable) hence also that $\theta(y)=\theta y$ for some constant $\theta$.

Conversely, we have the following (cf. [6]): if

$$
\lim _{x \rightarrow \infty} r(x)\left(\frac{U(x+y g(x))}{U(x)}-1\right)=\theta y,
$$

then this relation holds l.u. in $y$.

To conclude, we have the following theorem.

Theorem 1. Assume that $g \in S N$ and that $r \in \Gamma_{0}(g)$ with $r(x) \rightarrow \infty$.

(a) If Equation (1) or Equation (2) holds l.u. in $y$, then $\theta(x)=\theta x$ for some constant $\theta$.

(b) If Equation (1) or Equation (2) holds with $\theta(x)=\theta x$ for some constant $\theta$, then Equation (2) holds l.u. in $y$.

\subsection{Representation}

Three different ways to represent the functions satisfying Equation (1) follow.

\subsubsection{First Form}

For further use, let $A(x)=\int_{a}^{x} 1 / g(t) d t$. Clearly, we have

$$
A(x+y g(x))-A(x)=\int_{0}^{y} \frac{g(x)}{g(x+z g(x))} d z \rightarrow y
$$

1.u. in $y$. Note that $A(x)$ is an increasing function so that $f_{x}(y)=A(x+y g(x))-A(x)$ is an increasing function of $y$ for which $f_{x}(y) \rightarrow y$ as $x \rightarrow \infty$. As a consequence, the inverse function also satisfies $f_{x}^{-1}(y) \rightarrow y$. To calculate the inverse, we set

$$
f_{x}(y)=A(x+y g(x))-A(x)=t
$$

so that $x+y g(x)=A^{-1}(t+A(x))$ and

$$
y=f_{x}^{-1}(t)=\frac{A^{-1}(t+A(x))-A^{-1}(A(x))}{g(x)} .
$$

We conclude that

$$
\frac{A^{-1}(t+A(x))-A^{-1}(A(x))}{g(x)} \rightarrow t
$$

so that (replacing $A(x)$ by $x$ and $t$ by $y$ )

$$
\frac{A^{-1}(x+y)-A^{-1}(x)}{g\left(A^{-1}(x)\right)} \rightarrow y,
$$

l.u. in $y$.

Now, let $K(x):=W\left(A^{-1}(x)\right)$. We have (using l.u. convergence in the last step):

$$
\begin{aligned}
r(x)(K(A(x)+y)-K(A(x))) & =r(x)\left(W\left(A^{-1}(A(x)+y)\right)-W(x)\right) \\
& =r(x)\left(W\left(x+g(x) \frac{A^{-1}(A(x)+y)-x}{g(x)}\right)-W(x)\right) \\
& \rightarrow \theta y .
\end{aligned}
$$

It follows that

$$
r\left(A^{-1}(x)\right)(K(x+y)-K(x)) \rightarrow \theta y
$$


1.u. in $y$. Taking the integral $\int_{y=0}^{1}() d$.$y in Equation (3) we have$

$$
\int_{y=0}^{1} r\left(A^{-1}(x)\right)(K(x+y)-K(x)) d y \rightarrow \int_{0}^{1} \theta y d y
$$

or

$$
r\left(A^{-1}(x)\right) \int_{x}^{x+1} K(z) d z-r\left(A^{-1}(x)\right) K(x) \rightarrow \frac{\theta}{2} .
$$

We see that $K(x)$ is of the form

$$
\begin{aligned}
K(x) & =C+\int_{x}^{x+1} K(z) d z+\frac{C(x)}{r\left(A^{-1}(x)\right)} \\
& =L(x)+\frac{C(x)}{r\left(A^{-1}(x)\right)},
\end{aligned}
$$

where $C(x) \rightarrow C(=\theta / 2)$ and $L(x)=\int_{x}^{x+1} K(z) d z$. Note that

$$
r\left(A^{-1}(x) L^{\prime}(x)=r\left(A^{-1}(x)(K(x+1)-K(x)) \rightarrow \theta .\right.\right.
$$

Using $W(x)=K(A(x))$, we find that

$$
W(x)=T(x)+\frac{C^{\circ}(x)}{r(x)},
$$

where $C^{\circ}(x)=C(A(x)) \rightarrow C$ and $T(x)=L(A(x))$. Note that

$$
r(x) g(x) T^{\prime}(x)=r(x) L^{\prime}(A(x)) g(x) A^{\prime}(x)=r(x) L^{\prime}(A(x)) \rightarrow \theta .
$$

We prove the following result:

Theorem 2. Assume that $g \in S N$ and that $r \in \Gamma_{0}(g), r(x) \rightarrow \infty$.

(a) If Equation (1) holds with $\theta(x)=\theta x$, then $W(x)=\log U(x)$ is of the form

$$
W(x)=T(x)+\frac{C(x)}{r(x)}
$$

where $C(x) \rightarrow C$ and $r(x) g(x) T^{\prime}(x) \rightarrow \theta$.

(b) If $W(x)=T(x)+C(x) / r(x)$, where $C(x) \rightarrow 0$ and $r(x) g(x) T^{\prime}(x) \rightarrow \theta$, then Equation (1) holds with $\theta(y)=\theta y$.

Proof. The proof of (a) is given above. To prove (b), we have

$$
\begin{aligned}
& W(x+y g(x))-W(x) \\
= & T(x+y g(x))-T(x)+\frac{C(x+y g(x))}{r(x+y g(x))}-\frac{C(x)}{r(x)} .
\end{aligned}
$$

Clearly, we have

$$
r(x)(T(x+y g(x))-T(x))=y r(x) g(x) T^{\prime}(x+\beta g(x))
$$


for some $\beta \in(0, y)$. It follows that

$$
\begin{aligned}
r(x)(T(x+y g(x))-T(x)) & =y(\theta+o(1)) \frac{r(x) g(x)}{r(x+\beta g(x)) r(x+\beta g(x))} \\
& \rightarrow y \theta .
\end{aligned}
$$

For the second term, we have

$$
r(x)\left(\frac{C(x+y g(x))}{r(x+y g(x))}-\frac{C(x)}{r(x)}\right)=\frac{r(x)}{r(x+y g(x))} C(x+y g(x))-C(x) \rightarrow 0 .
$$

The result follows.

\section{Remark 1.}

1. In the special case where $g(x)=1$, we have

$$
\lim _{x \rightarrow \infty} r(x)(W(x+y)-W(x))=\theta y
$$

iff $W$ is of the form $W(x)=C+T(x)+\epsilon(x) / r(x)$ where $\epsilon(x) \rightarrow 0$ and $r(x) T^{\prime}(x) \rightarrow \theta$.

2. From Equation (1), it follows that

$$
\frac{r(x)}{U(x)}(U(x+y g(x))-U(x)) \rightarrow \theta y .
$$

The previous representation result shows that

$$
U(x)=T(x)+C(x) \frac{U(x)}{r(x)}
$$

where $r(x) g(x) T^{\prime}(x) \sim \theta U(x)$.

3. Using $U(x)=e^{W(x)}$, we also have that $U(x)=R(x) e^{C(x) / r(x)}$ where $R(x)=e^{T(x)}$. Note that

$$
r(x) g(x) \frac{R^{\prime}(x)}{R(x)}=r(x) g(x) T^{\prime}(x) \rightarrow \theta .
$$

\subsubsection{Second Form}

In Equation (3), we find that $r\left(A^{-1}(x)\right)(K(x+y)-K(x)) \rightarrow \theta y$, where $K(x)=W\left(A^{-1}(x)\right)$. Using logarithms, we get that

$$
\frac{K(\log x y)-K(x)}{L(x)} \rightarrow \theta \log y
$$

where $L(x)=r\left(A^{-1}(\log x)\right)$. From de Haan's theorem ([7], Theorem 3.7.3), we find that $K(\log x)$ can be written as

$$
K(\log x)=C+\theta L_{1}(x)+\int_{a}^{x} \theta L_{1}(t) t^{-1} d t,
$$

where $L_{1}(x) \sim L(x)$. It follows that

$$
K(x)=C+\theta L_{2}(x)+\theta \int_{a^{\circ}}^{x} L_{2}(t) d t,
$$

where $L_{2}(x)=L_{1}(\exp x) \sim r\left(A^{-1}(x)\right)$. 


\subsubsection{Third Form}

In [5], we found that relations of the form in Equation (1) hold with limit function $\theta(x)=0$. In that case, we have

$$
r(x)\left(\frac{U(x+y g(x))}{U(x)}-1\right) \rightarrow 0 .
$$

As usual, we assume that $g \in S N, r \in \Gamma_{0}(g)$ and $r(x) \rightarrow \infty$. From Theorem 3 in [5], we get the following representation:

$$
U(x)=\exp \left(c+\int_{0}^{x} f(t) d t\right)
$$

where $f$ satisfies $r(x) g(x) f(x) \rightarrow 0$.

\subsection{Sufficient Conditions}

In the next result, we assume that the $k$ th derivative of $U$ exists and we assume that

$$
h_{k}(x)=g(x) \frac{U^{(k)}(x)}{U^{(k-1)}(x)} \rightarrow 0,
$$

where $U^{(0)}(x)=U(x)$.

(a) If $k=1$, we have $U^{\prime}(x) / U(x)=\epsilon(x) / g(x)$ with $\epsilon(x) \rightarrow 0$ and

$$
\int_{x}^{x+y g(x)} \frac{U^{\prime}(z)}{U(z)} d z=\int_{x}^{x+y g(x)} \frac{\epsilon(z)}{g(z)} d z
$$

so that

$$
\log \frac{U(x+y g(x))}{U(x)}=\int_{0}^{y} \frac{\epsilon(x+z g(x)) g(x)}{g(x+z g(x))} d z \rightarrow 0
$$

and hence

$$
\frac{U(x+y g(x))}{U(x)} \rightarrow 1
$$

(b) If $k=2$, then we have

$$
\frac{U^{\prime \prime}(x)}{U^{\prime}(x)}=\frac{\epsilon(x)}{g(x)}
$$

and

$$
\int_{x}^{x+y g(x)} \frac{U^{\prime \prime}(z)}{U^{\prime}(z)} d z=\int_{x}^{x+y g(x)} \frac{\epsilon(z)}{g(z)} d z
$$

so that

$$
\begin{aligned}
& \log \frac{U^{\prime}(x+y g(x))}{U^{\prime}(x)} \\
&=\int_{0}^{y} \frac{\epsilon(x+z g(x))}{g(x+z g(x))} g(x) d z \rightarrow 0 .
\end{aligned}
$$

We find that

$$
\frac{U^{\prime}(x+y g(x))}{U^{\prime}(x)} \rightarrow 1
$$


Now, consider

$$
\begin{aligned}
U(x+y g(x))-U(x) & =\int_{x}^{x+y g(x)} U^{\prime}(z) d z \\
& =g(x) \int_{0}^{y} U^{\prime}(x+z g(x)) d z \\
& =g(x) U^{\prime}(x) \int_{0}^{y} \frac{U^{\prime}(x+z g(x))}{U^{\prime}(x)} d z \\
& \sim g(x) U^{\prime}(x) y
\end{aligned}
$$

and then

$$
\frac{U(x+y g(x))}{U(x)}-1 \sim h_{1}(x) y
$$

and thus Equation (1) holds with $r(x)=1 / h_{1}(x)$.

(c) If $k=3$, as before, we have

$$
\frac{U^{\prime \prime}(x+y g(x))}{U^{\prime \prime}(x)} \rightarrow 1
$$

and

$$
\begin{aligned}
U^{\prime}(x+y g(x))-U^{\prime}(x) & =g(x) U^{\prime \prime}(x) \int_{0}^{y} \frac{U^{\prime \prime}(x+z g(x))}{U^{\prime \prime}(x)} d z \\
& \sim g(x) U^{\prime \prime}(x) y .
\end{aligned}
$$

Further, we have

$$
U(x+y g(x))-U(x)=g(x) \int_{0}^{y} U^{\prime}(x+z g(x)) d z
$$

and

$$
\begin{aligned}
& U(x+y g(x))-U(x)-g(x) U^{\prime}(x) y \\
& \quad=g(x) \int_{0}^{y}\left(U^{\prime}(x+z g(x))-U^{\prime}(x)\right) d z \\
& \quad g^{2}(x) U^{\prime \prime}(x) \frac{y^{2}}{2}
\end{aligned}
$$

We conclude that

$$
\frac{U(x+y g(x))}{U(x)}-1-h_{1}(x) y \sim h_{1}(x) h_{2}(x) \frac{y^{2}}{2} .
$$

(d) In general, we get a result of the type

$$
\frac{U(x+y g(x))}{U(x)}-1-\sum_{i=1}^{k-1} \prod_{j=1}^{i} h_{j}(x) \frac{y^{i}}{i !} \sim \prod_{j=1}^{k} h_{j}(x) \frac{y^{k}}{k !} .
$$

As a special case, we can take $g(x)=1$ : if $U^{\prime \prime \prime}(x) / U^{\prime \prime}(x) \rightarrow 0$, then

$$
\frac{U(x+y)}{U(x)}-1-\frac{U^{\prime}(x)}{U(x)} y \sim \frac{U^{\prime \prime}(x)}{U(x)} \frac{y^{2}}{2} .
$$




\subsection{More Results}

Proposition 1. Suppose that $\bar{F}(x)=x^{-\alpha} L(x)$ where $L(\cdot)$ is a normalized slowly varying $(S V)$ function (that is, $\left.x L^{\prime}(x) / L(x) \rightarrow 0\right)$. Assume that $g(x)$ and $r(x)$ satisfy $g(x) / x \rightarrow 0$ and $r(x) g(x) / x \rightarrow \delta>0$. Then,

$$
r(x)\left(\frac{\bar{F}(x+y g(x))}{\bar{F}(x)}-1\right) \rightarrow-\alpha \delta y .
$$

Proof. We have $\bar{F}(x)=L(x) x^{-\alpha}$ and then

$$
\frac{\bar{F}(x+y g(x))}{\bar{F}(x)}=\frac{L(x+y g(x))}{L(x)} \times\left(1+y \frac{g(x)}{x}\right)^{-\alpha} .
$$

It follows that

$$
\begin{aligned}
\frac{\bar{F}(x+y g(x))}{\bar{F}(x)}-1 & =\frac{L(x+y g(x))}{L(x)} \times\left(\left(1+y \frac{g(x)}{x}\right)^{-\alpha}-1\right)+\frac{L(x+y g(x))}{L(x)}-1 \\
& =I(a)+I(b) .
\end{aligned}
$$

For $I(a)$, we have

$$
\frac{L(x+y g(x))}{L(x)}=\frac{L\left(x\left(1+y \frac{g(x)}{x}\right)\right)}{L(x)} \rightarrow 1,
$$

because $L$ is SV and $g(x) / x \rightarrow 0$. We also have

$$
\left(1+y \frac{g(x)}{x}\right)^{-\alpha}-1 \sim-\alpha y \frac{g(x)}{x},
$$

so that

$$
r(x)\left(\left(1+y \frac{g(x)}{x}\right)^{-\alpha}-1\right) \sim-\alpha y \frac{r(x) g(x)}{x} \rightarrow-\alpha \delta y .
$$

For the second term, we have

$$
\begin{aligned}
r(x)\left(\frac{L(x+y g(x))}{L(x)}-1\right) & =\frac{r(x)}{L(x)} \int_{x}^{x+y g(x)} L^{\prime}(t) d t \\
& =\frac{r(x)}{L(x)} \int_{x}^{x+y g(x)} \frac{t L^{\prime}(t)}{L(t)} \frac{L(t)}{t} d t \\
& =o(1) \frac{r(x) g(x)}{L(x)} \int_{0}^{y} \frac{L(x+\theta g(x))}{x+\theta g(x)} d \theta \\
& =o(1) \frac{r(x) g(x)}{x} .
\end{aligned}
$$

We conclude that

$$
r(x)\left(\frac{L(x+y g(x))}{L(x)}-1\right) \rightarrow 0 .
$$

Combining these results, we obtain the desired result.

Remark 2. The condition on $L(x)$ in the previous theorem is equivalent to the requirement that

$$
\frac{x f(x)}{\bar{F}(x)} \rightarrow \alpha,
$$

where $f(x)=F^{\prime}(x)$ is the density of $F$. 


\subsection{Examples}

\subsubsection{Example 1}

Assume that $U(x)=\exp x^{\beta}$ with $\beta>1$. We have

$$
\frac{U^{\prime}(x)}{U(x)}=\beta x^{\beta-1}
$$

and

$$
\frac{U^{\prime \prime}(x)}{U^{\prime}(x)}=\beta x^{\beta-1}+(\beta-1) x^{-1}
$$

Using $g(x)=x^{-\gamma}$, we find

$$
h_{1}(x)=g(x) \frac{U^{\prime}(x)}{U(x)}=\beta x^{\beta-\gamma-1}
$$

and

$$
h_{2}(x)=g(x) \frac{U^{\prime \prime}(x)}{U^{\prime}(x)}=\beta x^{\beta-\gamma-1}+(\beta-1) x^{-\gamma-1} .
$$

If $0<\beta-1<\gamma$, we find that $h_{1}(x) \rightarrow 0$ and $h_{2}(x) \rightarrow 0$. The results of this section show that

$$
\frac{U(x+y g(x))}{U(x)}-1 \sim h_{1}(x) y
$$

and Equation (1) holds with $r(x)=1 / h_{1}(x) \sim x^{\gamma+1-\beta} / \beta$.

\subsubsection{Example 2}

Assume that $U(x)=\exp x^{-\beta}$ with $\beta>0$. Clearly, we have

$$
\begin{aligned}
& \frac{U^{\prime}(x)}{U(x)}=-\beta x^{-\beta-1} \\
& \frac{U^{\prime \prime}(x)}{U^{\prime}(x)}=-\beta x^{-\beta-1}-(\beta+1) x^{-1} .
\end{aligned}
$$

We use $g(x)=x^{\gamma}$ and find

$$
\begin{aligned}
& h_{1}(x)=-\beta x^{\gamma-\beta-1} \\
& h_{2}(x)=h_{1}(x)-(\beta+1) x^{\gamma-1} .
\end{aligned}
$$

If $\gamma<\beta+1$, we have $h_{1}(x) \rightarrow 0$. If $\gamma<1$, we have $h_{1}(x) \rightarrow 0$ and $h_{2}(x) \rightarrow 0$. The results of the previous section show that

$$
\frac{U(x+y g(x))}{U(x)}-1 \sim h_{1}(x) y,
$$

and Equation (1) holds with $r(x)=1 / h_{1}(x) \sim-x^{\beta+1-\gamma} / \beta$.

\subsubsection{Example 3}

Assume that $U(x)=x^{\beta}$ where $\beta \neq 0$. We have

$$
h_{1}(x)=g(x) \frac{U^{\prime}(x)}{U(x)}=\beta \frac{g(x)}{x}
$$


and

$$
h_{2}(x)=g(x) \frac{U^{\prime \prime}(x)}{U^{\prime}(x)}=(\beta-1) \frac{g(x)}{x} .
$$

Taking $g \in S N$ and $r(x)=x / g(x)(\rightarrow \infty)$ we find

$$
r(x)\left(\frac{U(x+y g(x))}{U(x)}-1\right) \rightarrow \beta y .
$$

\subsubsection{Example 4}

Proposition 1 can be extended for some stable distributions. For instance, consider the density of an asymmetric stable distribution. The representation of such a stable density in the form of a convergent series is, for $0<\alpha<1$ and for any $x>0$ (see, e.g., [8]),

$$
q(x, \alpha, \rho)=\frac{1}{\pi} \sum_{n=1}^{\infty} \frac{(-1)^{n-1} \Gamma(\alpha n+1)}{n !} \sin (n \rho \pi) x^{-\alpha n-1} .
$$

Additionally, assume $x q^{\prime}(x, \alpha, \rho) / q(x, \alpha, \rho) \rightarrow \tau(\neq 0)$ as $x \rightarrow \infty$.

Let $g(x)$ and $r(x)$ be positive functions satisfying $g(x) / x \rightarrow 0$ and $r(x) g(x) / x \rightarrow \delta>0$.

Note that, for each $n>1$ and for $x$ large enough, we have, making use of $z-1 \sim \log z$ as $z \rightarrow 1$,

$$
\left(1+y \frac{g(x)}{x}\right)^{-\alpha n-1}-1 \sim-(\alpha n+1) \log \left(1+y \frac{g(x)}{x}\right) \sim-(\alpha n+1) y \frac{g(x)}{x} .
$$

Then, we have for $x$ large enough

$$
\begin{aligned}
& \frac{q(x+y g(x), \alpha, \rho)}{q(x, \alpha, \rho)}-1 \\
& =\frac{1}{\pi q(x, \alpha, \rho)} \sum_{n=1}^{\infty} \frac{(-1)^{n-1} \Gamma(\alpha n+1)}{n !} \sin (n \rho \pi) x^{-\alpha n-1}\left(\left(1+y \frac{g(x)}{x}\right)^{-\alpha n-1}-1\right) \\
& \sim y g(x) \frac{-1}{\pi q(x, \alpha, \rho)} \sum_{n=1}^{\infty}(\alpha n+1) \frac{(-1)^{n-1} \Gamma(\alpha n+1)}{n !} \sin (n \rho \pi) x^{-\alpha n-2} \\
& =y g(x) \frac{q^{\prime}(x, \alpha, \rho)}{q(x, \alpha, \rho)} .
\end{aligned}
$$

Hence, we have

$$
\lim _{x \rightarrow \infty} r(x)\left(\frac{q(x+y g(x), \alpha, \rho)}{q(x, \alpha, \rho)}-1\right)=y \delta \tau .
$$

\section{The Case $\alpha \neq 0$}

Now, suppose that $\alpha \neq 0$ and that

$$
\lim _{x \rightarrow \infty} r(x)\left(\frac{U(x+y g(x))}{U(x)}-e^{\alpha y}\right)=\theta(y),
$$

holds l.u. in $y$.

Equivalently, we have

$$
\lim _{x \rightarrow \infty} r(x)\left(\frac{e^{-\alpha y} U(x+y g(x))}{U(x)}-1\right)=e^{-\alpha y} \theta(y),
$$


and then (using $\log z \sim z-1$ )

$$
\lim _{x \rightarrow \infty} r(x)(W(x+y g(x))-W(x)-\alpha y)=\Omega(y),
$$

where $W(x)=\log U(x)$ and $\Omega(y)=e^{-\alpha y} \theta(y)$.

\subsection{The Limit}

In Equation (4), we replace $x$ by $x=t+z g(t)$ to find

$$
\lim _{x \rightarrow \infty} r(x)(W(t+z g(t)+y g(t+z g(t)))-W(t+z g(t))-\alpha y)=\Omega(y),
$$

and

$$
\begin{aligned}
\lim _{x \rightarrow \infty} & r(x) \\
& ((W(t+z g(t)+y g(t+z g(t)))-W(t)-\alpha(y+z))-(W(t+z g(t))-W(t)-\alpha z)) \\
& =\Omega(y) .
\end{aligned}
$$

The second term converges to $\Omega(z)$ and thus we have

$$
\begin{aligned}
& \lim _{x \rightarrow \infty} r(x) \\
& \quad\left(W\left(t+\left(z+y \frac{g(t+z g(t))}{g(t)}\right) g(t)\right)-W(t)-\alpha(y+z)\right) \\
& \quad=\Omega(z)+\Omega(y)
\end{aligned}
$$

or

$$
\begin{aligned}
& r(x)\left(W\left(t+\left(z+y \frac{g(t+z g(t))}{g(t)}\right) g(t)\right)-W(t)\right. \\
& \left.-\alpha\left(z+y \frac{g(t+z g(t))}{g(t)}\right)\right)+\alpha y\left(\frac{g(t+z g(t))}{g(t)}-1\right) \\
& \rightarrow \Omega(z)+\Omega(y) .
\end{aligned}
$$

By l.u. convergence, the first part converges to $\Omega(z+y)$ and then we have

$$
r(x) \alpha y\left(\frac{g(t+z g(t))}{g(t)}-1\right) \rightarrow \Omega(z)+\Omega(y)-\Omega(y+z) .
$$

Using the result of the previous subsection, we find that

$$
\alpha y \beta z=\Omega(z)+\Omega(y)-\Omega(y+z) .
$$

We propose a solution of the form $\Omega(z)=d x+c x^{2}$. The previous equation gives

$$
\alpha \beta y z=c z^{2}+c y^{2}-c\left(y^{2}+z^{2}+2 y z\right),
$$

and hence $\alpha \beta y z=2 c y z$ so that $c=\alpha \beta / 2$. We conclude that $\Omega(x)=d x+\alpha \beta x^{2} / 2$ and that $\theta(x)=$ $\left(d x+\alpha \beta x^{2} / 2\right) e^{\alpha x}$.

We conclude: 
Theorem 3. Suppose that $\alpha \neq 0$. If

$$
\lim _{x \rightarrow \infty} r(x)\left(\frac{U(x+y g(x))}{U(x)}-e^{\alpha y}\right)=\theta(y),
$$

holds l.u. in y, or equivalently if

$$
\lim _{x \rightarrow \infty} r(x)(W(x+y g(x))-W(x)-\alpha y)=\Omega(y)
$$

holds l.u. in $y$, then $g(x)$ satisfies Equation (2), $\Omega(x)=d x+\alpha \beta x^{2} / 2$ and $\theta(x)=\left(d x+\alpha \beta x^{2} / 2\right) e^{\alpha x}$.

\subsection{Special Case}

We assume that $W$ is differentiable and that $g(x) W^{\prime}(x) \rightarrow \alpha$.

In this case, we have

$$
\begin{aligned}
W(x+y g(x))-W(x) & =g(x) \int_{0}^{y} W^{\prime}(x+z g(x)) d z \\
& =\int_{0}^{y} \frac{g(x)}{g(x+z g(x))} g(x+z g(x)) W^{\prime}(x+z g(x)) d z \\
& \rightarrow \alpha y .
\end{aligned}
$$

Now, suppose in addition that $r(x)\left(g(x) W^{\prime}(x)-\alpha\right) \rightarrow \delta$ and that

$$
r(t)\left(\frac{g(x+\operatorname{tg}(x))}{g(x)}-1\right) \rightarrow \beta t .
$$

We have

$$
\begin{aligned}
& W(x+y g(x))-W(x)-\alpha y \\
&= g(x) \int_{0}^{y} W^{\prime}(x+\operatorname{tg}(x)) d t-\alpha y \\
&= \int_{0}^{y} g(x+\operatorname{tg}(x)) W^{\prime}(x+\operatorname{tg}(x)) \frac{g(x)}{g(x+\operatorname{tg}(x))} d t-\alpha y \\
&= \int_{0}^{y} g(x+\operatorname{tg}(x)) W^{\prime}(x+\operatorname{tg}(x))\left(\frac{g(x)}{g(x+\operatorname{tg}(x))}-1\right) d t \\
& \quad+\int_{0}^{y}\left(g(x+\operatorname{tg}(x)) W^{\prime}(x+\operatorname{tg}(x))-\alpha\right) d t .
\end{aligned}
$$

For the first integral, by assumption, we have

$$
r(t)\left(\frac{g(x+\operatorname{tg}(x))}{g(x)}-1\right) \rightarrow \beta t,
$$

or

$$
r(t) \frac{g(x+\operatorname{tg}(x))}{g(x)}\left(1-\frac{g(x)}{g(x+\operatorname{tg}(x))}\right) \rightarrow \beta t
$$

or

$$
r(t)\left(\frac{g(x)}{g(x+\operatorname{tg}(x))}-1\right) \rightarrow-\beta t .
$$


Since $r(x)\left(g(x) W^{\prime}(x)-\alpha\right) \rightarrow \delta$, we obtain

$$
\begin{aligned}
& r(x)(W(x+y g(x))-W(x)-\alpha y) \\
= & \int_{0}^{y} g(x+\operatorname{tg}(x)) W^{\prime}(x+\operatorname{tg}(x)) r(x)\left(\frac{g(x)}{g(x+\operatorname{tg}(x))}-1\right) d t \\
& +\int_{0}^{y} r(x)\left(g(x+\operatorname{tg}(x)) W^{\prime}(x+\operatorname{tg}(x))-\alpha\right) d t \\
\rightarrow & \alpha(-\beta) \frac{y^{2}}{2}+\delta y .
\end{aligned}
$$

\subsection{Representation Theorem}

Now, consider $Q(x)=W(x)+\alpha A(x)$, where $A(x)=\int_{a}^{x} 1 / g(t) d t$ as before. We prove above that

$$
A(x+y g(x))-A(x) \rightarrow y
$$

1.u. in $y$. If $g \in S N$ satisfies

$$
\lim _{x \rightarrow \infty} x\left(\frac{g(x+y g(x))}{g(x)}-1\right)=\beta y,
$$

then we also have

$$
\begin{aligned}
A(x+y g(x))-A(x)-y & =\int_{0}^{y}\left(\frac{g(x)}{g(x+z g(x))}-1\right) d z \\
& =-\int_{0}^{y} \frac{g(x)}{g(x+z g(x))}\left(\frac{g(x+z g(x))}{g(x)}-1\right) d z,
\end{aligned}
$$

so that

$$
r(x)(A(x+y g(x))-A(x)-y) \rightarrow-\beta \frac{y^{2}}{2} .
$$

Using $Q(x)=W(x)-\alpha A(x)$, we see that

$$
\begin{aligned}
& Q(x+y g(x))-Q(x) \\
= & W(x+y g(x))-W(x)-\alpha y \\
& -\alpha(A(x+y g(x))-A(x)-y) .
\end{aligned}
$$

Hence, using Equation (3),

$$
\begin{aligned}
& r(x)(Q(x+y g(x))-Q(x)) \\
= & r(x)(W(x+y g(x))-W(x)-\alpha y) \\
& -\alpha r(x)(A(x+y g(x))-A(x)-y) \\
\rightarrow & \Omega(y)+\alpha \beta \frac{y^{2}}{2}=\Psi(y)
\end{aligned}
$$

1.u. in $y$. As in the previous subsection, we conclude that

$$
r(x)(Q(x+y g(x))-Q(x)) \rightarrow \Psi(y)=\lambda y
$$

for some real number $\lambda$. The first representation of the previous subsection gives

$$
Q(x)=T(x)+\frac{C(x)}{r(x)}
$$


or

$$
W(x)=\alpha A(x)+T(x)+\frac{C(x)}{r(x)}
$$

where $C(x) \rightarrow C$ and $r(x) g(x) T^{\prime}(x) \rightarrow \lambda$.

Theorem 4. We have Equation (3) if and only if $W(x)$ is of the form

$$
W(x)=\alpha A(x)+T(x)+\frac{C(x)}{r(x)}
$$

where $C(x) \rightarrow C$ and $r(x) g(x) T^{\prime}(x) \rightarrow \lambda$

\subsection{More Results}

In our next result, we consider the function $h(x)=f(x) / \bar{F}(x)$, where $f$ is the density of $F$. We make the following assumptions about $h$ :

(a) $h \in S N$.

(b) $\quad r(x) h^{\prime}(x) / h^{2}(x) \rightarrow-\beta>0$, where $r(x) \rightarrow \infty, r(x) \in \Gamma_{0}(g)$ with $g(x)=1 / h(x)$.

Recall that $r \in \Gamma_{0}(g)$ means that $r(x+y g(x)) / r(x) \rightarrow 1$ as $x \rightarrow \infty$.

Lemma 1. If $(a)$ and $(b)$ hold, then

$$
r(x)\left(\frac{h(x+y g(x))}{h(x)}-1\right) \rightarrow-\beta y .
$$

Proof. We have

$$
h(x+y g(x))-h(x)=g(x) \int_{0}^{y} h^{\prime}(x+z g(x)) d z .
$$

Since $r(x) h^{\prime}(x) / h^{2}(x) \rightarrow-\beta>0$, we have that $h^{\prime}(x) \in \Gamma_{0}(g)$ and, using $g(x)=1 / h(x)$, we obtain that

$$
r(x)\left(\frac{h(x+y g(x))}{h(x)}-1\right)=\frac{r(x)}{h^{2}(x)} \int_{0}^{y} h^{\prime}(x+z g(x)) d z \rightarrow-\beta y .
$$

Now, we study the tail $\bar{F}(x)$.

Lemma 2. If $(a)$ and $(b)$ hold, then

$$
r(x)\left(\log \frac{\bar{F}(x+y g(x))}{\bar{F}(x)}+y\right) \rightarrow \beta \frac{y^{2}}{2} .
$$

Proof. Using $h(x)=f(x) / \bar{F}(x)$, we obtain that

$$
\int_{x}^{x+y g(x)} h(z) d z=\int_{x}^{x+y g(x)} \frac{f(z)}{\bar{F}(z)} d z,
$$

so that

$$
g(x) \int_{0}^{y} h(x+z g(x)) d z=-\log \frac{\bar{F}(x+y g(x))}{\bar{F}(x)} .
$$

It follows that $($ recall $g(x)=1 / h(x))$

$$
r(x) \int_{0}^{y}\left(\frac{h(x+z g(x))}{h(x)}-1\right) d z=-r(x)\left(\log \frac{\bar{F}(x+y g(x))}{\bar{F}(x)}+y\right)
$$


and using Lemma 1, it follows that

$$
r(x)\left(\log \frac{\bar{F}(x+y g(x))}{\bar{F}(x)}+y\right) \rightarrow \beta \frac{y^{2}}{2} .
$$

This proves the result.

Now, we arrive at the main result here.

Theorem 5. If (a) and (b) hold, then

$$
r(x)\left(\frac{\bar{F}(x+y g(x))}{\bar{F}(x)}-e^{-y}\right) \rightarrow \beta \frac{y^{2}}{2} e^{-y} .
$$

Proof. Using Lemma 2, we have

$$
r(x) \log e^{y} \frac{\bar{F}(x+y g(x))}{\bar{F}(x)} \rightarrow \beta \frac{y^{2}}{2} .
$$

Using $\log z \sim z-1$, it follows that

$$
r(x)\left(e^{y} \frac{\bar{F}(x+y g(x))}{\bar{F}(x)}-1\right) \rightarrow \beta \frac{y^{2}}{2},
$$

or

$$
r(x)\left(\frac{\bar{F}(x+y g(x))}{\bar{F}(x)}-e^{-y}\right) \rightarrow \beta \frac{y^{2}}{2} e^{-y}
$$

The previous theorem can be useful in extreme value theory as follows.

We assume that (a) and (b) hold and that $F$ is strictly increasing. We define $a_{n}$ by the equality $n \bar{F}\left(a_{n}\right)=1$. It is clear that $a_{n} \uparrow \infty$. In the result of Theorem 5 , we replace $x$ by $a_{n}$ to see that

$$
r\left(a_{n}\right)\left(n \bar{F}\left(a_{n}+y g\left(a_{n}\right)\right)-e^{-y}\right) \rightarrow \beta \frac{y^{2}}{2} e^{-y} .
$$

Now, we use $\log (z)+(1-z)=O(1)(1-z)^{2}$ and write

$$
\begin{aligned}
n \bar{F}\left(a_{n}+y g\left(a_{n}\right)\right) & =n \bar{F}\left(a_{n}+y g\left(a_{n}\right)\right)+n \log F\left(a_{n}+y g\left(a_{n}\right)\right)-n \log F\left(a_{n}+y g\left(a_{n}\right)\right) \\
& =O(1) n \bar{F}^{2}\left(a_{n}+y g\left(a_{n}\right)\right)-\log F^{n}\left(a_{n}+y g\left(a_{n}\right)\right) .
\end{aligned}
$$

Now, notice that

$$
r\left(a_{n}\right) n \bar{F}^{2}\left(a_{n}+y g\left(a_{n}\right)\right)=O(1) r\left(a_{n}\right) n \bar{F}^{2}\left(a_{n}\right)=O(1) \frac{r\left(a_{n}\right)}{n} .
$$

If $r\left(a_{n}\right) / n \rightarrow 0$, we obtain that

$$
r\left(a_{n}\right)\left(\log \bar{F}^{n}\left(a_{n}+y g\left(a_{n}\right)\right)+e^{-y}\right) \rightarrow-\beta \frac{y^{2}}{2} e^{-y},
$$

and hence also that

$$
r\left(a_{n}\right) \log e^{\exp -y} \bar{F}^{n}\left(a_{n}+y g\left(a_{n}\right)\right) \rightarrow-\beta \frac{y^{2}}{2} e^{-y},
$$


and

$$
r\left(a_{n}\right)\left(e^{\exp -y} \bar{F}^{n}\left(a_{n}+y g\left(a_{n}\right)\right)-1\right) \rightarrow-\beta \frac{y^{2}}{2} e^{-y}
$$

or

$$
r\left(a_{n}\right)\left(\bar{F}^{n}\left(a_{n}+y g\left(a_{n}\right)\right)-\exp -e^{-y}\right) \rightarrow-\beta \frac{y^{2}}{2} e^{-y} \exp -e^{-y} .
$$

It means that, if $X_{i}$ are independent and identically distributed random variables with distribution function $F$, then

$$
r\left(a_{n}\right)\left(P\left(\frac{M_{n}-a_{n}}{g\left(a_{n}\right)} \leq y\right)-\Lambda(y)\right) \rightarrow \Phi(y),
$$

where $M_{n}=\max \left(X_{1}, X_{2}, \ldots, X_{n}\right), \Lambda(y)=\exp -e^{-y}$ and $\Phi(y)=-\beta \frac{y^{2}}{2} e^{-y} \exp -e^{-y}$.

It means that $F$ is in the max-domain of attraction of the double exponential and the convergence rate is determined by $r\left(a_{n}\right)$.

\subsection{Examples}

\subsubsection{Example 1}

The following example is related to Theorem 5 .

Let $U(x)=\exp -x^{2}$ for $x>0$. Using $g(x)=1 /(2 x)$, we have $U(x) \in \Gamma_{-1}(g)$. Now, we consider the difference

$$
\frac{U(x+y g(x))}{U(x)}-e^{-y} .
$$

We have

$$
\begin{aligned}
\frac{U(x+y g(x))}{U(x)}-e^{-y} & =e^{-y-y^{2} g^{2}(x)}-e^{-y} \\
& =e^{-y}\left(e^{-y^{2} g^{2}(x)}-1\right) \\
& \sim-e^{-y} y^{2} g^{2}(x)
\end{aligned}
$$

and

$$
x^{2}\left(\frac{U(x+y g(x))}{U(x)}-e^{-y}\right) \rightarrow-\frac{1}{4} y^{2} e^{-y}
$$

3.5.2. Example 2

Let $U(x)=\exp x^{\beta}, \beta>1$. We have $W(x)=\log U(x)=x^{\beta}$ and $W^{\prime}(x)=\beta x^{\beta-1}$. Taking $g(x)=x^{1-\beta}$, we have

$$
g(x) W^{\prime}(x)=\beta
$$

As for $g(x)$, we have $g(x) / x \rightarrow 0$ and

$$
\frac{g(x+y g(x))}{g(x)}-1=\left(1+y \frac{g(x)}{x}\right)^{1-\beta}-1 \sim(1-\beta) y \frac{g(x)}{x} .
$$

Taking $r(x)=x / g(x)=x^{\beta}$, we have

$$
r(x)\left(\frac{g(x+y g(x))}{g(x)}-1\right) \rightarrow(1-\beta) y .
$$

The result of Section 3.2 shows that

$$
r(x)(W(x+y g(x))-W(x)-\beta y) \rightarrow \beta(\beta-1) \frac{y^{2}}{2},
$$


and then

$$
r(x)\left(\frac{U(x+y g(x))}{U(x)}-e^{\beta y}\right) \rightarrow \beta(\beta-1) \frac{y^{2}}{2} e^{\beta y}
$$

\section{Concluding Remarks}

In this paper, new results on the condition, for some functions $r$ and $g$,

$$
\lim _{x \rightarrow \infty} r(x)\left(\frac{U(x+y g(x))}{U(x)}-e^{\alpha y}\right)=\theta(y), \quad \forall y,
$$

where we assume that the convergence is l.u. in $y$, are presented. This limit generalizes the ones analyzed by Seneta [4] and Omey and Cadena [5], both of them being related to the monotony of functions in the Zygmund sense. Under this analysis, properties of $\theta(y)$ are described. Representations of the functions $U$ involved in this limit are provided.

Author Contributions: The authors have equally contributed to the writing, editing and style of the paper. All authors have read and agreed to the published version of the manuscript.

Funding: This research received no external funding.

Conflicts of Interest: The authors declare no conflict of interest.

\section{References}

1. Zygmund, A. Trigonometric Series, 2nd ed.; Cambridge University Press: Cambridge, UK, 1959; Volume 1.

2. Karamata, J. Sur un mode de croissance régulière des fonctions. Mathematica 1930, 4, 38-53.

3. Seneta, E. Regularly Varying Functions, Lecture Notes in Mathematics; Springer: Berlin/Heidelberg, Germany, 1976; Volume 508.

4. Seneta, E. Slowly varying functions in the Zygmund sense and generalized regular variation. J. Math. Anal. Appl. 2019, 475, 1647-1657. [CrossRef]

5. Omey, E.; Cadena, M. New results on slowly varying functions in the Zygmund sense. Proc. Jap. Acad. Ser. A 2019, accepted.

6. Omey, E. On the class gamma and related classes of functions. Publ. Inst. Math. 2013, 93, 1-18. [CrossRef]

7. Bingham, N.H.; Goldie, C.M.; Teugels, J.L. Regular Variation; Cambridge University Press: Cambridge, UK, 1987.

8. Uchaikin, V.V.; Zolotarev, V.M. Chance and Stability: Stable Distributions and Their Applications; De Gruyter: Utrecht, The Netherlands, 1999.

(C) 2020 by the authors. Licensee MDPI, Basel, Switzerland. This article is an open access article distributed under the terms and conditions of the Creative Commons Attribution (CC BY) license (http:/ / creativecommons.org/licenses/by/4.0/). 